\title{
Boundedness of multidimensional
}

\section{Hausdorff operator on Hardy-Morrey and Besov-Morrey spaces}

Belay Mitiku Damtew*

"Correspondence:

belaymida@yahoo.com Department of Mathematics,

Zhejiang Normal University, Jinhua,

Zhejiang 321004, P.R. China

\begin{abstract}
In this paper, we establish some boundedness conditions for the multidimensional Hausdorff operator on the homogeneous Hardy-Morrey and on the Besov-Morrey space, and we extend some results in the recent papers by Jia and Wang, and by Mazzucato, respectively. The main tool we implement in the study is the decomposition of the given function spaces in terms of atoms (smooth atoms for Besov-Morrey space) concentrated on dyadic cubes. The atomic decomposition of the classical Hardy space and Besov space is our study model, however, our case is a quite different one. Particularly, we will combine the Calderón reproducing formula with the atomic decomposition when we establish the boundedness of the Hausdorff operator on the Besov-Morrey space.
\end{abstract}

MSC: $42 \mathrm{~B} 35 ; 42 \mathrm{~B} 30$

Keywords: Hausdorff operator; boundedness; Hardy-Morrey space; Besov-Morrey space; atomic decomposition

\section{Introduction}

The one dimensional Hausdorff operator, for a fixed locally integrable function $\Phi$ on $(0, \infty)$, is defined by

$$
h_{\Phi}(f)(x)=\int_{0}^{\infty} \frac{\Phi(t)}{t} f\left(\frac{x}{t}\right) d t, \quad x \in \mathbb{R} .
$$

This operator has a long history in the study of mathematical analysis aiming to solve classical problems (see [1-3]).

The multidimensional Hausdorff operator on the Euclidean space $\mathbb{R}^{n}$, which was studied in [2] and [1], is defined by

$$
H_{\Phi, A}(f)(x)=\int_{\mathbb{R}^{n}} \frac{\Phi(y)}{|y|^{n}} f(A(y) x) d y .
$$

Here, in (1.1) and in the sequel, we fix $\Phi \in L_{\text {loc }}^{1}\left(\mathbb{R}^{n}\right)$ and assume that $A=A(y)=\left(a_{i j}\right)_{i, j=1}^{n}=$ $\left(a_{i j}(y)\right)_{i, j=1}^{n}$ is an $n \times n$ nonsingular matrix almost everywhere in the support of $\Phi$. The entries $a_{i j}(y)$ of the matrix $A$ are measurable functions of $y$.

(c) The Author(s) 2016. This article is distributed under the terms of the Creative Commons Attribution 4.0 International License (http://creativecommons.org/licenses/by/4.0/), which permits unrestricted use, distribution, and reproduction in any medium, provided you give appropriate credit to the original author(s) and the source, provide a link to the Creative Commons license, and indicate if changes were made. 
We denote

$$
\|A\|=\max _{1 \leq j \leq n} \sum_{i=1}^{n}\left|a_{i j}\right|
$$

to be the operator $\ell$-norm of the operator in an $n$ dimensional linear space defined by the matrix $A$ in the corresponding canonical basis (see [2]).

The boundedness of $h_{\Phi}(f)$ as well as its high dimensional extensions in various function spaces, mainly on Lebesgue and Hardy spaces, has been studied by many authors, for example, [1-5] and [6] are among many others. The aim of this paper is to establish some boundedness conditions for the multidimensional Hausdorff operator on the homogeneous Hardy-Morrey space and the Besov-Morrey space, since these spaces are 'upgrade version' of the Lebesgue space and the Hardy space and they play significant roles in the theory of harmonic analysis and partial differential equations (see [7-10] and [11]).

The Hardy-Morrey space $H \mathcal{M}_{q}^{p}(q \leq 1)$, as a generalization of the classical Morrey spaces $\mathcal{M}_{q}^{p}(q>1)$ and Hardy spaces $H^{p}(p \leq 1)$, was introduced by Jia and Wang [7]. Among many features of $H \mathcal{M}_{q}^{p}$, particularly Jia and Wang establish the decomposition of $H \mathcal{M}_{q}^{p}$ in terms of atoms concentrated on dyadic cubes, which have the same cancelation properties as the atoms in the classical Hardy space. Another space, one that we are also interested in, is the Besov-Morrey space $\mathcal{N}_{p q r}^{s}$ for $1 \leq q \leq p<\infty, 1 \leq r \leq \infty$ and $s \in \mathbb{R}$. This space was originally introduced by Kozono and Yamazaki [8] in order to investigate time-local solutions of the Navier-Stokes equations with the initial data in Besov-Morrey spaces. Later, Mazzucato [9] studied the atomic and molecular decompositions on BesovMorrey spaces. Sawano and Tanaka [10] further developed a theory of decompositions in Besov-Morrey spaces $\mathcal{N}_{p q r}^{s}$ and Triebel-Lizorkin-Morrey spaces $\mathcal{E}_{p q r}^{s}$ with $0<q \leq p<\infty$, $0<r \leq \infty, s \in \mathbb{R}$. The homogeneous Besov-Morrey space $\mathcal{N}_{p q r}^{s}$ is a function space whose norm is obtained by replacing the $L^{p}$-norm in the definition of the Besov space with the $\mathcal{M}_{q}^{p}$-norm. Any function in the Besov-Morrey space has a decomposition in terms of atoms supported on dyadic cubes. These atoms have the same smoothness and cancelation properties as those of the classical Besov spaces [11]. Thus, in our study, we will invoke the atomic decomposition characterization for both Hardy-Morrey space and Besov-Morrey space. Besides the atomic decomposition, the Calderón reproducing formula is another useful tool when we treat the boundedness of $H_{\Phi, A}(f)$ on the Besov-Morrey space.

The following definitions and previously obtained results (Theorem A and Theorem B) about the function spaces we consider are crucial in our study.

Definition 1.1 For $p$ and $q$ satisfying $1 \leq q \leq p<\infty$, the homogeneous Morrey space $\mathcal{M}_{q}^{p}$ is defined as

$$
\mathcal{M}_{q}^{p}\left(\mathbb{R}^{n}\right)=\left\{f \in L_{\mathrm{loc}}^{q}\left(\mathbb{R}^{n}\right):\|f\|_{\mathcal{M}_{q}^{p}\left(\mathbb{R}^{n}\right)}=\sup _{\substack{x_{0} \in \mathbb{R}^{n} \\ R>0}}\left|B\left(x_{0}, R\right)\right|^{1 / p-1 / q}\|f\|_{L^{q}\left(B\left(x_{0}, R\right)\right)}<\infty\right\},
$$

where $B\left(x_{0}, R\right)$ is the closed ball of $\mathbb{R}^{n}$ with center $x_{0}$ and radius $R$.

We note that the Morrey space describes local regularity more precisely than $L^{p}$ and can be seen as a complement to $L^{p}$-spaces. In fact one easily checks $L^{p}=\mathcal{M}_{p}^{p}$ and $L^{p} \subset \mathcal{M}_{q}^{p}$, if $1 \leq q \leq p<\infty$. Jia and Wang in [7] give the definition of Hardy-Morrey space as follows. 
Definition 1.2 For $0<q \leq p<\infty$, the Hardy-Morrey space $H \mathcal{M}_{q}^{p}$ is defined as

$$
H \mathcal{M}_{q}^{p}\left(\mathbb{R}^{n}\right)=\left\{f \in S^{\prime} / P:\|f\|_{H \mathcal{M}_{q}^{p}\left(\mathbb{R}^{n}\right)}=\left\|\sup _{s>0}\left|\phi_{s} * f\right|\right\|_{\mathcal{M}_{q}^{p}\left(\mathbb{R}^{n}\right)}<\infty\right\}
$$

where $\phi_{s}(x)=s^{-n} \phi\left(s^{-1} x\right), s>0$ for $\phi \in S\left(\mathbb{R}^{n}\right)$ with $\int \phi d x=1$, and $P$ is the set of all polynomials in $n$ variables. From the above definition, we note that:

(i) $H \mathcal{M}_{q}^{p}=\mathcal{M}_{q}^{p}$ for $1<q \leq p<\infty$,

(ii) $H^{p}=H \mathcal{M}_{p}^{p} \subset H \mathcal{M}_{q}^{p}$ for $q \leq p<\infty$, and in particular, $H \mathcal{M}_{1}^{1}=H^{1}$.

As stated in [7], the Hardy-Morrey space $H \mathcal{M}_{q}^{p}(q \leq 1)$ generalizes the Hardy space $H^{q}(q \leq 1)$. Fixing $q=1$ in (1.3), we see that the space $H \mathcal{M}_{1}^{p}\left(\mathbb{R}^{n}\right)$ for $1 \leq p<\infty$ is a generalization of the Hardy space $H^{1}$. Therefore, we may adapt the atomic decomposition method used in [1] and [6] for treating the Hardy space to study the newly introduced Hardy-Morrey space $H \mathcal{M}_{1}^{p}(1 \leq p<\infty)$ to deal with the boundedness of the Hausdorff operator (1.1). However, we must modify the main step since the decompositions of $H^{1}$ and $H \mathcal{M}_{1}^{p}$ are essentially different when $p>1$ (see the following definition). We also note that $H \mathcal{M}_{1}^{p}(1 \leq p<\infty)$ is a Banach space with the norm in (1.3).

Definition 1.3 Let $j \in \mathbb{Z}$ and $k \in \mathbb{Z}^{n}$. The set

$$
Q=Q_{j k}=\left\{x \in \mathbb{R}^{n}: 2^{-j} k_{i} \leq x_{i} \leq 2^{-j}\left(k_{i}+1\right), i=1,2, \ldots, n\right\}
$$

is called a dyadic cube, with sides parallel to the coordinate axes. For fixed $j$, the collection $\left\{Q_{j k}: k \in \mathbb{Z}^{n}\right\}$ tiles the whole space and the cubes are pairwise disjoint. Each cube $Q=Q_{j k}$ is uniquely identified by its length $\ell(Q)=2^{-j}$ and a preferred corner $x_{Q}=2^{-j} k$.

We remark that, for a dyadic cube $J$ and $q=1,(1.2)$ can also be written as

$$
\|f\|_{\mathcal{M}_{1}^{p}} \approx \sup _{J: \text { dyadic }}|J|^{1 / p-1}\|f\|_{L^{1}(J)}
$$

This equivalent norm of $\mathcal{M}_{1}^{p}$ will play a pivotal role in our study.

Definition 1.4 For a dyadic cube $Q$, a function $a_{Q}$ is called a $(p, 1)_{L}$-atom of $H \mathcal{M}_{1}^{p}(1 \leq$ $p<\infty), L \in \mathbb{N} \cup\{0\}$, if the following support, boundedness, and cancelation conditions are satisfied:

(i) $\operatorname{supp} a_{Q} \subset 3 Q$;

(ii) $\left\|a_{Q}\right\|_{L^{p}} \leq|Q|^{1 / p-1}$;

(iii) $\int_{\mathbb{R}^{n}} x^{\alpha} a_{Q}(x) d x=0, \quad|\alpha| \leq L$,

where $L \geq[n(1-1 / p)],[x]$ is the Gauss function, $3 Q$ is a cube concentric with $Q$ of side length $3 \ell(Q)$ and $|Q|$ stands for the volume of $Q$.

The next theorem is directly adapted from [7] when $q=1$, and is important in the proof for one of the main theorems (Theorem 2.1). In Theorem A and in the sequel, we make use of (1.4) to get the norm of the sequence of complex numbers $s=\left\{s_{Q}: Q\right.$ dyadic $\}$. 
Theorem A ([7]) For $1 \leq p<\infty$, let $\left\{a_{Q}: Q\right.$ dyadic $\}$ be a collection of $(p, 1)_{L}$-atoms and $s=\left\{s_{Q}: Q\right.$ dyadic $\}$ be a sequence of complex numbers with

$$
\|s\|_{p, 1}=\sup _{J}\left(\frac{1}{|J|}\right)^{1-1 / p} \sum_{Q \subset J}|Q|^{1-1 / p}\left|s_{Q}\right|<\infty .
$$

The sum

$$
f=\sum_{Q: \text { dyadic }} s_{Q} a_{Q}
$$

converges in $S^{\prime} / P$, where $f \in H \mathcal{M}_{1}^{p}$ satisfying $\|f\|_{H \mathcal{M}_{1}^{p}} \leq C\|s\|_{p, 1}$ for some constant $C=$ $C(n, p)$.

Conversely, every function $f \in H \mathcal{M}_{1}^{p}$ has the atomic decomposition (1.7) in $S^{\prime} / P$ with $\|s\|_{p, 1} \leq C\|f\|_{H \mathcal{M}_{1}^{p}}$ for some $C=C(n, p)$.

The other important function space in harmonic analysis is the Besov-Morrey space, which has many applications in the study of nonlinear partial differential equations, for example see in [8-10], and [11].

Again, our interest here is to study the boundedness of $n$ dimensional Hausdorff operators on the homogeneous Besov-Morrey space. To this end, as we mentioned before, we need to use the smooth atomic decomposition of $\mathcal{N}_{p q r}^{s}$, and this will be implemented in the Fourier transform side and by using the results in [12] and [13] for classical Besov spaces. Let $S$ be the Schwartz space on $\mathbb{R}^{n}$. For $\varphi \in S\left(\mathbb{R}^{n}\right)$, as in [14], its Fourier transform is denoted by $\hat{\varphi}$ and its inverse Fourier transform is denoted by $\check{\varphi}$. Take $\varphi_{0} \in S\left(\mathbb{R}^{n}\right)$ with $\varphi_{0} \geq 0$ and

$$
\varphi_{0}(x)= \begin{cases}1, & |x| \leq 1 \\ 0, & |x| \geq 2 \\ \text { smooth, } & \text { elswhere }\end{cases}
$$

Now define $\varphi(x)=\varphi_{0}(x)-\varphi_{0}(2 x)$ and set $\varphi_{j}(x)=\varphi\left(2^{-j} x\right)$ for all $j \in \mathbb{Z}$. Then $\left\{\varphi_{j}, j \in \mathbb{Z}\right\}$ is a homogeneous smooth dyadic resolution of unity in $\mathbb{R}^{n}$, namely $\sum_{j \in \mathbb{Z}} \varphi_{j}(x)=1$ for all $x \in \mathbb{R}^{n}$. Moreover, let $\psi_{j}=\check{\varphi}_{j}$. Thus, with independence of the choice of $\psi_{j}$, we give the Fourier analytic definition of $\mathcal{N}_{p q r}^{s}$ as follows.

Definition 1.5 Let $s \in \mathbb{R}, 1 \leq q \leq p<\infty$, and $1 \leq r \leq \infty$. We say that $f \in S^{\prime} / P$ belongs to the space $\mathcal{N}_{p q r}^{s}\left(\mathbb{R}^{n}\right)$ if

$$
\|f\|_{\mathcal{N}_{p q r}^{s}\left(\mathbb{R}^{n}\right)}= \begin{cases}\left\{\sum _ { j \in \mathbb { Z } } \left(2^{j s}\left\|\psi_{j} * f\right\|_{\left.\left.\mathcal{M}_{q}^{p}\right)^{r}\right\}^{1 / r}<\infty,}\right.\right. & 1 \leq r<\infty, \\ \sup _{j \in \mathbb{Z}} 2^{j s}\left\|\psi_{j} * f\right\|_{\mathcal{M}_{q}^{p}<\infty,} & r=\infty .\end{cases}
$$

One can see that in [8] $\mathcal{N}_{p q r}^{s}$ is a Banach space under the norm (1.8). This space generalizes the classical homogeneous Besov space. In particular, $\mathcal{N}_{p p r}^{s}=\dot{B}_{p r}^{s}$.

The atomic decomposition of $K$-times continuously differentiable functions in this space can be obtained in [9]. 
Definition 1.6 Let $s \in \mathbb{R} ; K, L+1 \in N_{0}=\mathbb{N} \cup\{0\}$. A function $a_{Q}$ is called an $(s, p)_{K, L}$-atom if the following support, smoothness, and cancelation conditions are satisfied for some dyadic cube $Q$ :

(i) $\operatorname{supp} a_{Q} \subset 3 Q$;

(ii) $\left|\partial^{\alpha} a_{Q}(x)\right| \leq C|Q|^{\frac{s}{n}-\frac{1}{p}-\frac{|\alpha|}{n}}, \quad|\alpha| \leq K$;

(iii) $\int_{\mathbb{R}^{n}} x^{\alpha} a_{Q}(x) d x=0, \quad|\alpha| \leq L$,

where $K \geq([s]+1)$ and $L \geq \max \{[-s+n / p],-1\}$.

Definition 1.7 [11] Let $1 \leq q \leq p<\infty$ and $1 \leq r \leq \infty$. Then $\mathbf{n}_{p q r}\left(\mathbb{R}^{n}\right)$ is the collection of all sequences $s=\left\{s_{Q}: Q\right.$ dyadic $\}$ such that

$$
\|s\|_{\mathbf{n}_{p q r}\left(\mathbb{R}^{n}\right)}=\left\{\sum_{j \in \mathbb{Z}}\left(\sup _{\substack{J: \text { dyadic } \\ \ell(J) \geq 2^{-j}}}\left(\frac{1}{|J|}\right)^{1-q / p} \sum_{\substack{Q \subset J \\ \ell(Q)=2^{-j}}}|Q|^{1-q / p}\left|s_{Q}\right|^{q}\right)^{r / q}\right\}^{1 / r}<\infty .
$$

Remark (norm equivalence) For $f \in \mathcal{N}_{p q r}^{s}\left(\mathbb{R}^{n}\right)$ with atomic decomposition $f=\sum_{Q} s_{Q} a_{Q}$, $\left\{s_{Q}\right\}$ satisfies

$$
\|f\|_{\mathcal{N}_{p q r}^{s}\left(\mathbb{R}^{n}\right)} \approx\left(\left\|\sum_{\ell(Q)=2^{-j}} s_{Q} \tilde{\chi}_{Q}\right\|_{\mathcal{M}_{q}^{p}}\right)_{l^{r}}
$$

where $\tilde{\chi}_{Q}=|Q|^{-1 / p} \chi_{Q}$ is an $L^{p}$-normalized characteristic function of the cube $Q$.

Theorem $\mathbf{B}$ ([11]) Suppose $s \in \mathbb{R}, 1 \leq q \leq p<\infty$ and $r \in[1, \infty]$. Then $f \in \mathcal{N}_{p q r}^{s}\left(\mathbb{R}^{n}\right)$ has the atomic decomposition

$$
f=\sum_{Q: \text { dyadic }} s_{Q} a_{Q}
$$

in $S^{\prime} / P$, where $a_{Q}$ is a collection of $(s, p)_{K, L}$-atoms. Moreover, the sequence of complex numbers $s=\left\{s_{Q}\right\}$ satisfies $\|s\|_{\mathbf{n}_{p q r}} \leq C\|f\|_{\mathcal{N}_{p q r}^{s}}$ for some $C=C(n, p, q, r)$.

The proof of this theorem is obtained in [9].

To obtain the reverse direction of the second part of Theorem B we need the molecular decomposition characterization of the space $\mathcal{N}_{p q r}^{s}\left(\mathbb{R}^{n}\right)$, which is less important in this paper. The analogous definition of $(s, p)_{K, L, M}$-molecule for $M>0$, can be found in [9] and [14].

In this paper, we use the notation $A \preceq B$ to mean that there is a positive constant $C$ (may vary at each appearance) independent of all essential variables such that $A \leq C B$. We also use the notation $A \simeq B$ to mean that there are positive constants $C_{1}$ and $c_{1}$ independent of all essential variables such that $c_{1} B \leq A \leq C_{1} B$. 


\section{Main results}

Now we are in a position to state our main results. The proof will be given in Section 3.

Theorem 2.1 For any $1 \leq p<\infty$, the Hausdorff operator (1.1) is bounded on the HardyMorrey space $H \mathcal{M}_{1}^{p}$, i.e.

$$
\left\|H_{\Phi, A}(f)\right\|_{H \mathcal{M}_{1}^{p}\left(\mathbb{R}^{n}\right)} \preceq C_{1}\|f\|_{H \mathcal{M}_{1}^{p}\left(\mathbb{R}^{n}\right)},
$$

provided

$$
C_{1}=\int_{\mathbb{R}^{n}} \frac{|\Phi(y)|}{|y|^{n}}\left\|A^{-1}(y)\right\|^{n-n / p}\left|\operatorname{det} A^{-1}(y)\right|^{1 / p} d y<\infty .
$$

For $n=1$ we state the following boundedness result as a corollary to the above theorem.

Corollary 2.2 Let $1 \leq p<\infty$. If $\Phi \in L^{1}(0, \infty)$, then the operator

$$
h_{\Phi}(f)(x)=\int_{0}^{\infty} \frac{\Phi(t)}{t} f\left(\frac{x}{t}\right) d t
$$

is bounded on the Hardy-Morrey space $H \mathcal{M}_{1}^{p}$. Precisely we have

$$
\left\|h_{\Phi}(f)\right\|_{H \mathcal{M}_{1}^{p}(\mathbb{R})} \preceq\left(\int_{0}^{\infty}|\Phi(t)| d t\right)\|f\|_{H \mathcal{M}_{1}^{p}(\mathbb{R})} .
$$

Theorem 2.3 For $s \in \mathbb{R}, 1 \leq q \leq p<\infty$ and $1 \leq r \leq \infty$, the Hausdorffoperator $H_{\Phi, A}(f)(x)=$ $\int_{\mathbb{R}^{n}} \frac{\Phi(y)}{|y|^{n}} f(A(y) x) d y$ is bounded on the Besov-Morrey space $\mathcal{N}_{p q r}^{s}\left(\mathbb{R}^{n}\right)$, i.e.

$$
\left\|H_{\Phi, A}(f)\right\|_{\mathcal{N}_{p q r}^{s}\left(\mathbb{R}^{n}\right)} \preceq \int_{\mathbb{R}^{n}} \frac{|\Phi(y)|}{|y|^{n}} \beta(y)^{-1}\|A(y)\|^{s-n(1 / p-1 / q)}\left|\operatorname{det} A^{-1}(y)\right|^{1 / q} d y\|f\|_{\mathcal{N}_{p q r}^{s}\left(\mathbb{R}^{n}\right)},
$$

where

$$
\beta(y)=\left(\|A(y)\|\left\|A^{-1}(y)\right\|\right)^{s-n / p-|\alpha|} .
$$

\section{Proofs of main results}

Proof of Theorem 2.1 We prove this theorem using the atomic characterization of the Hardy-Morrey space. As stated in Theorem A, every function $f \in H \mathcal{M}_{1}^{p}$ has the atomic decomposition

$$
f=\sum_{Q} s_{Q} a_{Q}
$$

in $S^{\prime} / P$, where

$$
\|f\|_{H \mathcal{M}_{1}^{p}} \simeq\|s\|_{p, 1}
$$

in which each $a_{Q}$ is a $(p, 1)_{L}$-atom as in Definition 1.4, and the sequence of complex numbers $s=\left\{s_{Q}\right\}$ satisfies (1.6). Thus, by (1.3) and the Minkowski integral inequality, we have 


$$
\begin{aligned}
& \left\|H_{\Phi, A}(f)\right\|_{H \mathcal{M}_{1}^{p}\left(\mathbb{R}^{n}\right)} \\
& =\left\|\sup _{s>0}\left|\phi_{s} *\left(H_{\Phi, A} f\right)\right|\right\|_{\mathcal{M}_{1}^{p}\left(\mathbb{R}^{n}\right)} \\
& =\sup _{x_{0} \in \mathbb{R}^{n}}\left|B\left(x_{0}, R\right)\right|^{1 / p-1}\left|\sup _{s>0}\right| \phi_{s} * \int_{\mathbb{R}^{n}} \frac{\Phi(y)}{|y|^{n}} f(A(y) x) d y \mid \|_{L^{1}\left(B\left(x_{0}, R\right)\right)} \\
& =\sup _{x_{0} \in \mathbb{R}^{n}}\left|B\left(x_{0}, R\right)\right|^{1 / p-1} \int_{B\left(x_{0}, R\right)} \sup _{s>0}\left|\phi_{s} * \int_{\mathbb{R}^{n}} \frac{\Phi(y)}{|y|^{n}} f(A(y) x) d y\right| d x \\
& \leq \int_{\mathbb{R}^{n}} \frac{|\Phi(y)|}{|y|^{n}} \sup _{x_{0} \in \mathbb{R}^{n}}\left|B\left(x_{0}, R\right)\right|^{1 / p-1} \int_{B\left(x_{0}, R\right)} \sup _{s>0}\left|\phi_{s} * f(A(y) \cdot) x\right| d x d y \\
& =\int_{\mathbb{R}^{n}} \frac{|\Phi(y)|}{|y|^{n}}\left\|A^{-1}(y)\right\|^{n-n / p}\left|\operatorname{det} A^{-1}(y)\right|^{1 / p}\|g(A(y) \cdot)\|_{H \mathcal{M}_{1}^{p}\left(\mathbb{R}^{n}\right)} d y,
\end{aligned}
$$

where

$$
g(A(y) \cdot)=\left\|A^{-1}(y)\right\|^{n / p-n}\left|\operatorname{det} A^{-1}(y)\right|^{-1 / p} f(A(y) \cdot) .
$$

Hence, we have

$$
\|g(A(y) \cdot)\|_{H \mathcal{M}_{1}^{p}\left(\mathbb{R}^{n}\right)}=\left\|\sum_{Q} s_{Q}\right\| A^{-1}(y)\left\|^{n / p-n}\left|\operatorname{det} A^{-1}(y)\right|^{-1 / p} a_{Q}(A(y) \cdot)\right\|_{H \mathcal{M}_{1}^{p}\left(\mathbb{R}^{n}\right)} .
$$

In the above formula, we note

$$
a_{Q_{y}}(\cdot)=\left\|A^{-1}(y)\right\|^{n / p-n}\left|\operatorname{det} A^{-1}(y)\right|^{-1 / p} a_{Q}(A(y) \cdot) .
$$

Therefore, to complete the proof of the theorem, it remains to show that

$$
\left\|a_{Q_{y}}(\cdot)\right\|_{H \mathcal{M}_{1}^{p}\left(\mathbb{R}^{n}\right)} \preceq 1
$$

uniformly for all atoms $a_{Q}$ and $y \in \mathbb{R}^{n}$. To this end, it suffices to show that $a_{Q_{y}}(x)$ is a $(p, 1)_{L}$-atom. By Definition 1.4 we have $\operatorname{supp} a_{Q} \subset 3 Q$, where

$$
Q=Q_{j k}=\prod_{i=1}^{n}\left[2^{-j} k_{i}, 2^{-j}\left(k_{i}+1\right)\right], \quad \text { and set } \quad 3 Q=\prod_{i=1}^{n}\left[2^{-j}\left(k_{i}-1\right), 2^{-j}\left(k_{i}+2\right)\right] .
$$

Without loss of generality, assume that $a_{Q}(A(y) x) \neq 0$, so that there exists $u \in 3 Q$ such that $u=A(y) x \in 3 Q$. Thus

$$
x=A^{-1}(y) u \in A^{-1}(3 Q)=3 A^{-1}(Q) .
$$

It yields

$$
\operatorname{supp} a_{Q}(A(y) x) \subset 3\left(A^{-1} Q\right),
$$


where $A^{-1} Q=A^{-1}(y) Q$ is a parallelepiped with vertices that are images of the vertices of the cube $Q$ after left multiplying by the matrix $A^{-1}$. We now enlarge the domain $A^{-1} Q$ to $Q_{A^{-1}}$, where $Q_{A^{-1}}$ is the least circumscribed cube that contains $A^{-1} Q$ (see $[2,5]$ ). Thus it follows that $3\left(A^{-1} Q\right) \subset 3 Q_{A^{-1}}$.

Since $a_{Q_{y}}(x)=a_{Q}(A(y) x)$ up to a constant independent of $y$, we have

$$
\operatorname{supp} a_{Q_{y}} \subset 3 Q_{A^{-1}},
$$

and from the side length relations of $Q_{A^{-1}}$ and $Q$, we easily obtain

$$
\left|Q_{A^{-1}}\right| \simeq\left\|A^{-1}\right\|^{n}|Q|
$$

Obviously, $a_{\mathrm{Q}}$ satisfies the cancelation condition (1.5)(iii). Finally, we show the size condition of $a_{Q_{y}}$ as follows:

$$
\begin{aligned}
\left\|a_{Q_{y}}(\cdot)\right\|_{L^{p}\left(\mathbb{R}^{n}\right)} & =\left(\left.\left.\int_{\mathbb{R}^{n}}\left|a_{Q}(A(y) x)\left\|A^{-1}(y)\right\|^{n / p-n}\right| \operatorname{det} A^{-1}(y)\right|^{-1 / p}\right|^{p} d x\right)^{1 / p} \\
& =\left\|A^{-1}(y)\right\|^{n / p-n}\left|\operatorname{det} A^{-1}(y)\right|^{-1 / p}\left(\int_{\mathbb{R}^{n}}\left|a_{Q}(A(y) x)\right|^{p} d x\right)^{1 / p} \\
& \leq\left\|A^{-1}(y)\right\|^{n / p-n}|Q|^{1 / p-1} \\
& =\left(\left\|A^{-1}(y)\right\|^{n}|Q|\right)^{1 / p-1} \\
& \preceq\left|Q_{A^{-1}}\right|^{1 / p-1} .
\end{aligned}
$$

Thus, we conclude that $a_{Q_{y}}$ is a $(p, 1)_{L}$-atom and hence prove the theorem.

The proof of Corollary 2.2 coincides with the proof of the main theorem when $n=1$.

Next, we continue by investigating the boundedness of $H_{\Phi, A}$ on the Besov-Morrey space.

Proof of Theorem 2.3 We prove this theorem by combining the Calderón's reproducing formula with some construction on a dyadic cube $Q_{j k}$ and the smooth atomic decomposition of the Besov-Morrey space.

First, we make a construction on $a_{Q}$ and $s_{Q}$ with respect to the dyadic cube $Q$ based on the corresponding Besov spaces as in [12] and [15].

Let $\left\{\psi_{j}, j \in \mathbb{Z}\right\} \subset S\left(\mathbb{R}^{n}\right)$ be a smooth dyadic resolution of unity as described in Section 2, and pick a smooth, radial function $\theta \in S\left(\mathbb{R}^{n}\right)$ satisfying:

(i) $\operatorname{supp} \theta \subset\left\{x \in \mathbb{R}^{n}:|x| \leq 1\right\}$,

(ii) $\hat{\theta}(\xi) \geq c>0 \quad$ if $1 / 2 \leq|\xi| \leq 2$,

(iii) $\int_{\mathbb{R}^{n}} x^{\alpha} \theta(x) d x=0 \quad$ for all $|\alpha| \leq L$,

such that the Calderón reproducing formula

$$
\sum_{j \in \mathbb{Z}} \hat{\theta}\left(2^{j} \xi\right) \hat{\psi}\left(2^{j} \xi\right)=1
$$


holds for all $\xi \in \mathbb{R}^{n} \backslash\{0\}$. Define $\theta_{j}(x)=2^{j n} \theta\left(2^{j} x\right)$. Then, for $f \in S^{\prime} / P$, we can write

$$
f=\sum_{j \in \mathbb{Z}} \theta_{j} * \psi_{j} * f
$$

By the definition of the cube $Q_{j k}$, we further write

$$
\begin{aligned}
f(x) & =\sum_{j \in \mathbb{Z}} \sum_{Q: \ell(Q)=2^{-j}} \int_{Q_{j k}} \theta_{j}(x-y)\left(\psi_{j} * f\right)(y) d y \\
& =\sum_{j \in \mathbb{Z}} 2^{j n} \sum_{Q: \ell(Q)=2^{-j}} \int_{Q_{j k}} \theta\left(2^{j}(x-y)\right)\left(\psi_{j} * f\right)(y) d y .
\end{aligned}
$$

For $Q=Q_{j k}$, define

$$
s_{Q}=C|Q|^{1 / p-s / n} \sup _{y \in Q}\left|\psi_{j} * f(y)\right|
$$

and

$$
a_{Q}=\frac{1}{s_{Q}} \int_{Q} \theta_{j}(x-y)\left(\psi_{j} * f\right)(y) d y \quad\left(s_{Q} \neq 0\right) .
$$

Now, using Definition 1.7 with the assumption that $Q$ is contained in $J$, and the proof used in [9], we can show that the sequence of complex numbers $s=\left\{s_{Q}\right\}$ of (3.1) satisfies

$$
\|s\|_{\mathbf{n}_{p q r}\left(\mathbb{R}^{n}\right)}=\left\{\sum_{j \in \mathbb{Z}}\left(\sup _{\substack{J: \text { dyadic } \\ \ell(J) \geq 2^{-j}}}\left(\frac{1}{|J|}\right)^{1-q / p} \sum_{\substack{Q \subset J \\ \ell(Q)=2^{-j}}}|Q|^{1-q / p}\left|s_{Q}\right|^{q}\right)^{r / q}\right\}^{1 / r} \leq C\|f\|_{\mathcal{N}_{p q r}^{s}\left(\mathbb{R}^{n}\right)},
$$

for some constant $C$ independent of $f$.

$a_{Q}$ in (3.2) satisfies the smoothness condition (ii) of (1.9), and depending on the requirements of $\theta$ it also satisfies condition (i) and (iii) of (1.9). Therefore $a_{Q}$ is an $(s, p)_{K, L}$-atom.

Next, we take any function $f \in \mathcal{N}_{p q r}^{s}\left(\mathbb{R}^{n}\right)$ with the above atomic decomposition,

$$
f=\sum_{Q} s_{Q} a_{Q} \quad \text { in } S^{\prime} / P
$$

and

$$
\|f\|_{\mathcal{N}_{p q r}^{s}\left(\mathbb{R}^{n)}\right.} \simeq\left(\left\|\sum_{\ell(Q)=2^{-j}} s_{Q} \tilde{X}_{Q}\right\|_{\mathcal{M}_{q}^{p}}\right)_{l^{r}} .
$$

Thus, using Definition 1.5 and the Minkowski integral inequality in the following computations, we have

$$
\begin{aligned}
& \left\|H_{\Phi, A}(f)\right\|_{\mathcal{N}_{p q r}^{s}\left(\mathbb{R}^{n}\right)} \\
& =\left\{\sum_{j \in \mathbb{Z}}\left(2^{j s}\left\|\psi_{j} *\left(H_{\Phi, A} f\right)\right\|_{\mathcal{M}_{q}^{p}}\right)^{r}\right\}^{1 / r}
\end{aligned}
$$




$$
\begin{aligned}
& =\left\{\sum_{j \in \mathbb{Z}}\left(2^{j s q} \sup _{\substack{x_{0} \in \mathbb{R}^{n} \\
R>0}}\left|B\left(x_{0}, R\right)\right|^{q / p-1} \int_{B\left(x_{0}, R\right)}\left|\psi_{j} * \int_{\mathbb{R}^{n}} \frac{\Phi(y)}{|y|^{n}} f(A(y) x) d y\right|^{q} d x\right)^{r / q}\right\}^{1 / r} \\
& \leq \int_{\mathbb{R}^{n}} \frac{|\Phi(y)|}{|y|^{n}}\left\{\sum_{j \in \mathbb{Z}}\left(2^{j s q} \sup _{\substack{x_{0} \in \mathbb{R}^{n} \\
R>0}}\left|B\left(x_{0}, R\right)\right|^{q / p-1} \int_{B\left(x_{0}, R\right)}\left|\psi_{j} * f(A(y) \cdot) x\right|^{q} d x\right)^{r / q}\right\}^{1 / r} d y \\
& =\int_{\mathbb{R}^{n}} \frac{|\Phi(y)|}{|y|^{n}} \beta(y)^{-1}\|A(y)\|^{s-n(1 / p-1 / q)}\left|\operatorname{det} A^{-1}(y)\right|^{1 / q} d y\|\beta(y) h(A(y) \cdot)\|_{\mathcal{N}_{p q r}^{s}\left(\mathbb{R}^{n}\right)^{\prime}}
\end{aligned}
$$

where

$$
h(A(y) \cdot)=\|A(y)\|^{h(1 / p-1 / q)-s}\left|\operatorname{det} A^{-1}(y)\right|^{-1 / q} f(A(y) \cdot) .
$$

Furthermore, by the definition, we obtain

$$
\begin{aligned}
& \|\beta(y) h(A(y) \cdot)\|_{\mathcal{N}_{p q r}^{s}\left(\mathbb{R}^{n}\right)} \\
& \quad=\left\|\sum_{Q} s_{Q} \beta(y)\right\| A(y)\left\|^{n(1 / p-1 / q)-s}\left|\operatorname{det} A^{-1}(y)\right|^{-1 / q} a_{Q}(A(y) \cdot)\right\|_{\mathcal{N}_{p q r}^{s}\left(\mathbb{R}^{n}\right)},
\end{aligned}
$$

where

$$
a_{Q_{y}}(\cdot)=\beta(y)\|A(y)\|^{n(1 / p-1 / q)-s}\left|\operatorname{det} A^{-1}(y)\right|^{-1 / q} a_{Q}(A(y) \cdot) .
$$

In the same way as in the proof of Theorem 2.1, for any atom $a_{Q}$ and fixed $y \in \mathbb{R}^{n}$, we need to show that the function $a_{Q y}$ is an $(s, p)_{K, L}$-atom satisfying Definition 1.6.

Together with the cancelation condition of $\theta$, it is straightforward to verify that $a_{Q_{y}}$ given in (3.3) satisfies the cancelation condition for every $y \in \mathbb{R}^{n}$. Based on the construction of $a_{Q}$ over $Q$ in (3.2) and the requirement of the support condition of $\theta$, we see that $a_{Q}$ is supported in 3Q. Since $a_{Q y}(x)$ in (3.3) is a constant multiple of $a_{Q}(A(y) x)$, then without loss of generality, we may use a similar discussion made in the proof of Theorem 2.1 for the support condition. Thus we conclude that

$$
\operatorname{supp} a_{Q_{y}} \subset 3 Q_{A^{-1}}
$$

where $a_{Q}$ is the one in (3.3) and $Q_{A^{-1}}$ is the smallest circumscribed cube as discussed in Theorem 2.1.

Finally by using (3.2) and the definition of $\theta_{j}$, we see the smoothness condition as follows:

$$
\begin{aligned}
& \left|\partial^{\alpha} a_{Q_{y}}(x)\right| \\
& \quad=\left|\partial^{\alpha}\left(\beta(y)\|A(y)\|^{n(1 / p-1 / q)-s}\left|\operatorname{det} A^{-1}(y)\right|^{-1 / q} a_{Q}(A(y) x)\right)\right| \\
& \quad=\beta(y)\|A(y)\|^{n(1 / p-1 / q)-s}\left|\operatorname{det} A^{-1}(y)\right|^{-1 / q}\left|\partial^{\alpha} a_{Q}(A(y) x)\right| \\
& \quad \leq \beta(y)\|A(y)\|^{n(1 / p-1 / q)-s}\|A(y)\|^{n / q} \frac{1}{s_{Q}} \int_{Q}\left|\partial^{\alpha}\left(\theta_{j}(A(y) x-y)\right) \|\left(\psi_{j} * f\right)(y)\right| d y \\
& \quad=\frac{1}{C} \beta(y)\|A(y)\|^{n / p-s}|Q|^{s / n-1 / p}\left(\int_{Q}\left|\partial^{\alpha}\left(2^{j n} \theta\left(2^{j}(A(y) x-y)\right)\right) \|\left(\psi_{j} * f\right)(y)\right| d y\right)
\end{aligned}
$$




$$
\begin{aligned}
& \cdot\left(\sup _{y \in Q}\left|\left(\psi_{j} * f\right)(y)\right|\right)^{-1} \\
\leq & \frac{1}{C} \beta(y)\|A(y)\|^{n / p-s}|Q|^{s / n-1 / p} 2^{j(n+|\alpha|)}\|A(y)\|^{|\alpha|} \int_{Q}\left|\left(\partial^{\alpha} \theta\right)\left(2^{j}(A(y) x-y)\right)\right| d y \\
\leq & C \beta(y)\|A(y)\|^{n / p-s+|\alpha|}|Q|^{s / n-1 / p-1-|\alpha| / n}|Q| \\
= & C\left(\|A(y)\|\left\|A^{-1}(y)\right\|\right)^{s-n / p-|\alpha|}\|A(y)\|^{n / p-s+|\alpha|}|Q|^{s / n-1 / p-|\alpha| / n} \\
= & C\left(\left\|A^{-1}(y)\right\|^{n}|Q|\right)^{s / n-1 / p-|\alpha| / n} \\
= & C\left|Q_{A^{-1}}\right|^{s / n-1 / p-|\alpha| / n} .
\end{aligned}
$$

Thus, $a_{Q y}(x)$ is an $(s, p)_{K, L}$-atom.

Therefore, we complete the proof of the theorem.

\section{Competing interests}

The author declare that there is no conflict of interests regarding the publication of this paper.

\section{Author's information}

Belay Mitiku Damtew is a Doctor in the field of harmonic analysis, applied mathematics.

\section{Acknowledgements}

This research work was supported by NSF of China (no. 11471288, 11671363, 11371136) and NSF of Zhejiang, China (no. LQ17A010001).

Received: 21 June 2016 Accepted: 14 November 2016 Published online: 22 November 2016

\section{References}

1. Chen, J, Fan, D, Li, J: Hausdorff operators on function spaces. Chin. Ann. Math., Ser. B 33, 537-556 (2012)

2. Lerner, AK, Liflyand, E: Multidimensional Hausdorff operators on the real Hardy spaces. J. Aust. Math. Soc. 83, 79-86 (2007)

3. Liflyand, E: Hausdorff operators on Hardy spaces. Eurasian Math. J. 4, 101-141 (2013)

4. Chen, J, Fan, D, Lin, X, Ruan, J: The fractional Hausdorff operators on the Hardy spaces $H^{p}\left(\mathbb{R}^{n}\right)$. Anal. Math. 42, 1-17 (2016)

5. Chen, J, Fan, D, Zhang, C: Boundedness of Hausdorff operators on some product Hardy type spaces. Appl. Math. J. Chin. Univ. Ser. A 27, 114-126 (2012)

6. Ruan, J, Fan, D: Hausdorff operators on the power weighted Hardy spaces. J. Math. Anal. Appl. 433, 31-48 (2016)

7. Jia, H, Wang, H: Decomposition of Hardy-Morrey spaces. J. Math. Anal. Appl. 354, 99-110 (2009)

8. Kozono, H, Yamazaki, M: Semilinear heat equations and the Navier-Stokes equation with distributions in the new function spaces as initial data. Commun. Partial Differ. Equ. 19(5-6), 959-1014 (1994)

9. Mazzucato, AL: Decomposition of Besov-Morrey spaces. Contemp. Math. 320, 279-294 (2003)

10. Sawano, Y, Tanaka, H: Decompositions of Besov-Morrey spaces and Triebel-Lizorkin-Morrey spaces. Math. Z. 257, 871-905 (2007)

11. Wang, H: Decomposition for Morrey type Besov-Triebel spaces. Math. Nachr. 282(5), 774-787 (2009)

12. Frazier, M, Jawerth, B: Decomposition of Besov spaces. Indiana Univ. Math. J. 34, 777-799 (1985)

13. Triebel, H: Theory of Function Spaces. Birkhäuser, Basel (1983)

14. Fu, J, Xu, J: Characterizations of Morrey type Besov and Triebel-Lizorkin spaces with variable exponents. J. Math. Anal. Appl. 381, 280-298 (2011)

15. Drihem, D: Atomic decomposition of Besov spaces with variable smoothness and integrability. J. Math. Anal. Appl. 389, 15-31 (2012) 